\title{
Assessment of 10 years of maize pedigree breeding for European corn borer tolerance and high-yielding combining ability
}

\author{
Antoine Panouilléa*, Pierre Anglade ${ }^{b}$, Armand Boyat ${ }^{c}$, Brigitte Gouesnard ${ }^{\mathrm{c}}$, \\ Jean-Claude Vible ${ }^{b}$, Michel Dupin ${ }^{a}$ \\ ${ }^{a}$ Unité expérimentale du maïs, Inra, 40390 Saint-Martin de Hinx, France \\ b Unité de recherches de zoologie, Inra, BP 81, 33883 Villenave d'Ornon, France \\ ${ }^{c}$ Unité de formation et de recherches de génétique et amélioration des plantes, Inra, 34130 Mauguio, France
}

(Received 3 March 1997; accepted 9 June 1998)

\begin{abstract}
A multitrait pedigree breeding system including evaluation for European corn borer (Ostrinia nubilalis Hbn.) tolerance and other agronomic traits (yield, earliness, stalk lodging) was used for 16 years to create inbred lines from very different temperate germplasms. The ultimate evaluation of the 63 inbred lines was made in comparison with stable known references. The results allowed us to classify this material into three tolerance classes to the insect and demonstrated the efficacy of the method. High-yielding combining ability might be associated with earliness, lodging tolerance and good insect tolerance. The value of some early flint European materials and of Argentinian sources was discussed to improve European corn borer tolerance. (@ Inra/Elsevier, Paris.)
\end{abstract}

Zea mays L. / inbred lines / pedigree breeding / corn borer tolerance / high yielding combining ability

Résumé - Bilan de dix années d'évaluation de lignées de maïs créées par sélection généalogique multicaractère combinant tolérance à la pyrale et aptitude à la combinaison pour le rendement. Pour associer la tolérance à la pyrale $(O$. nubilalis $\mathrm{Hbn}$.) aux caractères agronomiques habituellement sélectionnés, des programmes de création et d'évaluation de lignées du maïs ont été développés par les laboratoires Inra du Maïs (Montpellier, Saint-Martin de Hinx, Bordeaux). En pépinière et dans les essais d'évaluation, la tolérance est exprimée par une note de dégâts à maturité corrigée pour les différences de précocité du matériel. L'utilisation de lignées étalons éprouvées et reconnues stables permet le classement de lignées en trois groupes de tolérance. La méthode de sélection généalogique repose sur une sélection simultanée par niveaux indépendants pour les deux caractères. Des essais de valeur hybride sur différents testeurs complètent les tris en valeur propre. Du matériel tolérant est obtenu dans les différentes sources utilisées. Toutefois, le

Communicated by André Gallais (Gif-sur-Yvette)

* Correspondence and reprints 
matériel précoce européen et des sources argentines paraissent plus favorables à l'obtention de lignées tolérantes. Ces programmes ont permis l'obtention d'une soixantaine de lignées à forte aptitude à la combinaison pour le rendement et à bons niveaux de tolérance à la pyrale, avec des gains de précocité et de résistance à la verse. (@ Inra/Elsevier, Paris.)

\section{Zea mays L. / lignées pures / sélection généalogique / tolérance à la pyrale / aptitude à la combinaison}

\section{INTRODUCTION}

At the beginning of the $1970 \mathrm{~s}$ the maize acreage in Europe hugely increased, mainly with early varieties in the northern areas. These crops were progressively invaded by European corn borer (E.C.B.), Ostrinia nubilalis Hbn., populations which normally develop only one generation per year. In France, the first noticeable occurrence of the borer on new maize crops in the Beauce area was recorded in 1966 [2]. With the lack of natural parasites, both the levels of the E.C.B. population and the damage on the susceptible varieties rose quickly. Control with granulated insecticides was initiated as a temporary alternative measure and management of borer populations using variety resistance began to be considered.

At this time, in Iowa, Dicke and Guthrie developed a laboratory production of E.C.B. egg masses for field infestation and obtained several inbred lines with antibiosis resistance to leaf-feeding [6]. It was shown that the survival rate of the first instar larvae depended on the deterrent and toxic effects of the amount of D.I.M.B.O.A. in the whorl-leaf tissues [11]. Moreover, a late line B52 was found to present a high degree of resistance to sheath-collar feeding after anthesis due to a distinct mechanism [7].

However, in north-European ecological conditions, field observations established that E.C.B. oviposition occurred at the end of the late-whorl stage [8] so that the earlier larvae fed primarily on the tissues of the tassel climbing up in the whorl and the later larvae on sheath and collar tissues. In these conditions, the leaf-feeding resistant NorthAmerican lines were of little interest as resource for early material breeding. Moreover, the authors failed in transferring the B52 characters of sheath- collar resistance to adapted early European lines (unpublished results), probably as the consequence of heavy linkage between these characters and unfavourable characters systematically contraselected.

Consequently, attention was turned to tolerance, the 'third basis of resistance', defined by Painter [13] as the ability of the plant to withstand, to a marked degree, insect host infestation. On maize, tolerance depends on the role played by the plant in reducing the general boring in the stalk which disturbs the transfer of nutrients to the ear and in the strengthening of tissues which avoids stalk breakage. Tolerance may also be considered as the ability, when the tolerated insect is present, to reduce yield losses due to the borer compared to the losses in a susceptible genotype [3].

Research projects on tolerance were initiated in collaboration with the international working group I.W.G.O./I.O.B.C. Many lines were exchanged among most European countries and evaluated by the same procedures in various environments. Sources of tolerance were discovered. The heritability of tolerance characters was demonstrated [9]. Field experiments showed the efficacy of tolerant lines in reducing in their hybrids the yield losses mainly attributed to a reduction of the weight of individual kernels (unpublished data). Programmes were developed to introduce tolerance into conventional breeding for early and mid-early materials adapted to European conditions. The aim of this paper was: 1) to present the methods used in a pedigree breeding for E.C.B. tolerance and highyielding combining ability; 2) to summarise and compare in the same way the annual evaluations of more than 60 inbred lines released for 10 years in succession from 1987 to 1996 ; 3) to compare the value of different germplasm resources to improve E.C.B. tolerance. 


\section{MATERIALS AND METHODS}

\subsection{Basic genetic materials}

The pedigree selection programmes successively developed for 16 years used sources covering a large range of maize variability: early European populations and lines, Plata Argentina germplasm, Lancaster and Stiff Stalk dent lines, for instance. These sources were primarily selected for agronomic requirements. The breeding programmes were developed either from single crosses and back-crosses or from composites and openpollinated populations.

\subsection{Pedigree breeding}

\subsubsection{E.C.B. Tolerance evaluation}

The plants were artificially infested at the late-whorl stage of plant growth with a low level of $1-2 \mathrm{egg}$ masses per plant only, making estimations of different degrees of tolerance easier. Near physiological maize maturity, plant damage was rated according to a discontinuous rating scale $(1=$ little damage, $2=$ broken tassel, $3=2+$ broken upper leaves, $4.5=$ broken upper stalk, $9=$ broken stalk under the ear or dropped ear) [1].
This method was used either for screening in the nursery or for evaluating the lines in experimental trials. For these trials, around 20 to 50 lines were allotted to experimental designs according to earliness with ten plants per plot and four replicates, including 8-10 tolerant and susceptible standards. The plot means of individual tolerance ratings per plant (TR) were calculated and compared by means of variance analysis. Then the regression of TR data on silking dates or on a 1 (early)6 (late) precocity index (PI) related to the plant growth stage at egg-hatching was studied. Figure I shows, as an example, how the final TR of a line may be estimated by the algebraic value of the difference, $D$, between observed data and its expected value in the regression. The final TR could also be expressed by adding the D algebraic value to the general mean of the trial. These tests were repeated for 2 years.

\subsubsection{Breeding system}

To achieve simultaneous breeding for tolerance (TOL) and agronomic conventional traits (AGR), an original breeding system was developed (table I). First, S0 plants were screened for tolerance only. A $25 \%$ selection rate eliminated most of the susceptible plants. The selection in nursery among $\mathrm{S} l$ families used the independent culling level method keeping the best families with ratings above the respective thresholds for both criteria. Then, the advanced generations were developed in nursery without any borer selection pressure. In the
Figure 1. Example of presentation of the tolerance ratings obtained in an annual experiment. Regression of the tolerance ratings (TR) on the 1-6 precocity index (PI) related to the plant growth stage at egg hatching. Note the distances ( $\mathrm{D}+$ or $\mathrm{D}-$ ) between the actual data for a given line and its expected value according to the regression.

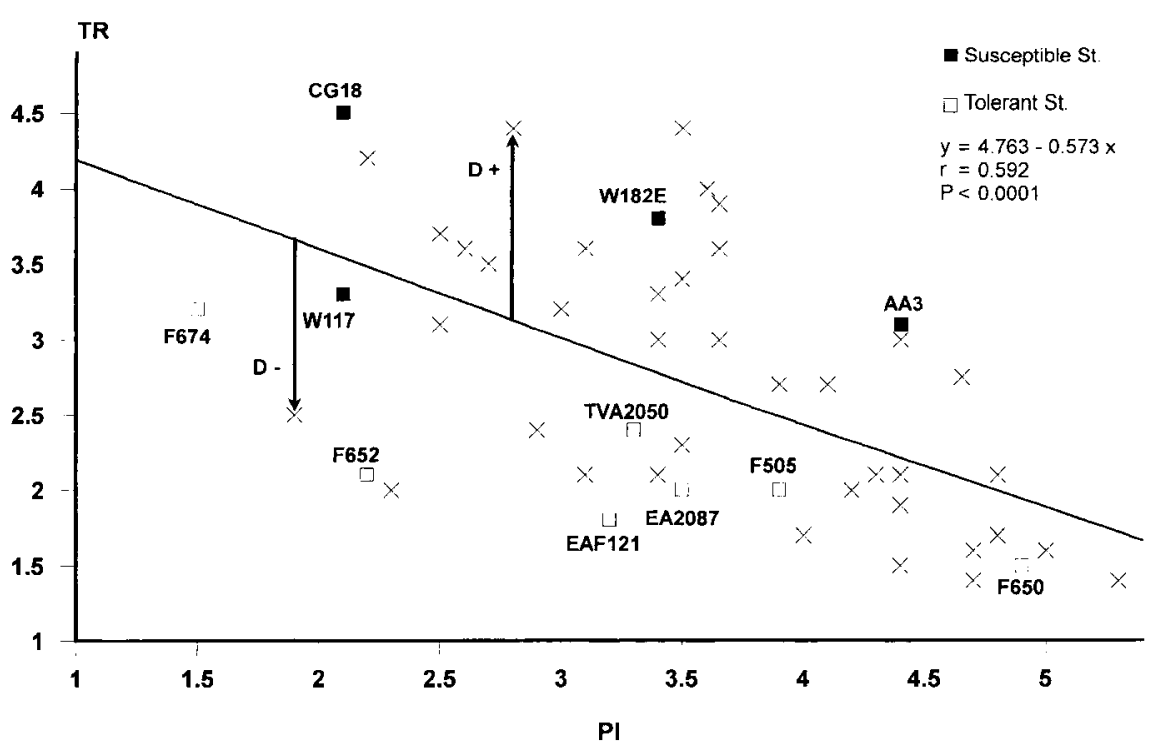


Table I. Schema of pedigree selection including ECB tolerance evaluation.

\begin{tabular}{|c|c|c|c|c|c|}
\hline Years & Generations & & $\begin{array}{l}\text { Value per se } \\
\text { Materials and criteri }\end{array}$ & & $\begin{array}{l}\text { Hybrid value } \\
\text { Materials and criteria }\end{array}$ \\
\hline 1 & $\mathrm{~S} 0 \rightarrow \mathrm{S} 1$ & So plants & TOL & & \\
\hline 2 & & S1 lines & $\mathrm{TOL}+\mathrm{AGR}$ & & \\
\hline 3 & & Repeat & & & \\
\hline 4 & $\mathrm{~S} 1 \rightarrow \mathrm{S} 2$ & & & & TC seed production \\
\hline 5 & & & & & Yield test $\mathrm{S} 1 / \mathrm{TC}$ hybrids AGR \\
\hline 6 & $\mathrm{~S} 2 \rightarrow \mathrm{S} 3$ & $\mathrm{~S} 2$ bulks test & TOL & S2 nursery AGR & TC seed production \\
\hline 7 & $\mathrm{~S} 3 \rightarrow \mathrm{S} 4$ & Repeat & & S3 nursery AGR & Yield test S2/TC hybrids AGR \\
\hline 8 & $\mathrm{~S} 4 \rightarrow \mathrm{S} 5$ & & & S4 nursery AGR & TC seed production \\
\hline 9 & $\mathrm{~S} 5 \rightarrow \mathrm{S} 6$ & S5 lines test & TOL & S5 nursery AGR & Yield test S4/TC hybrids AGR \\
\hline 10 & $\mathrm{~S} 6 \rightarrow \mathrm{S} 7$ & Repeat & & S6 nursery AGR & Repeat \\
\hline
\end{tabular}

TOL $=$ European corn borer tolerance evaluation; $\mathrm{AGR}=$ evaluation of agronomic traits; $\mathrm{TC}=$ test-crosses.

mean time, screenings of S2-S3 families on bulks were independently carried out in distinct field trials for tolerance and for agronomic value in test-crosses. Finally, the performances of the S5 lines were evaluated over 2 years for per se tolerance in experimental plots. On the other hand, the agronomic value on test-crosses (earliness, stalk lodging and yield) was compared over 2 years in an experimental network to reference lines crossed on the same testers. These references (REF) relevant to the concerned germplasm of the line were supposed to be the best ones of the moment. The lines to be released presented an yield equal or above the reference.

\subsection{Method for summarising 10 years of results}

The results were presented in two ways. First, an attempt was made to draw up a common list of the 63 released lines ordered according to their tolerance level

Table II. Characteristics of E.C.B tolerance standards for final evaluation of inbred lines developed in multitrait breeding programmes (1986-1995).

\begin{tabular}{|c|c|c|c|c|c|c|c|}
\hline \multirow{2}{*}{$\begin{array}{l}\text { Tolerance } \\
\text { classes }\end{array}$} & \multirow{2}{*}{$\begin{array}{l}\text { Standard } \\
\text { lines }\end{array}$} & \multirow{2}{*}{$\begin{array}{l}\text { Pedigrees or } \\
\text { origins }\end{array}$} & \multirow[t]{2}{*}{ Silking ${ }^{2}$} & \multicolumn{4}{|c|}{ Tolerance ratings ${ }^{3}$} \\
\hline & & & & M & $\mathrm{CL}$ & FT & $\mathrm{VC} \%$ \\
\hline \multirow[t]{4}{*}{ Tolerant } & TVA 2050 & Trnava/Slovakia & +0.5 & \multicolumn{2}{|c|}{$1.63 \pm 0.12$} & a & 8.0 \\
\hline & F 888 & $\mathrm{Sel} / \mathrm{ETO}(\mathrm{M}) \mathrm{C} 9^{1}$ & +5.0 & \multicolumn{2}{|c|}{$1.79 \pm 0.24$} & $a b$ & 14.9 \\
\hline & EA 2087 & Sel/OP Azpeitia, Spain & -3.0 & \multicolumn{2}{|c|}{$2.08 \pm 0.16$} & $a b$ & 9.3 \\
\hline & F 652 & $\mathrm{~F} 2 \times \mathrm{BGR} 477^{1}$ & -4.0 & \multicolumn{2}{|c|}{$2.24 \pm 0.25$} & $\mathrm{~b}$ & 13.6 \\
\hline \multirow[t]{3}{*}{ Susceptible } & F 804 & FS14 synthetic ${ }^{1}$ & +2.0 & \multirow{3}{*}{\multicolumn{2}{|c|}{$\begin{array}{l}4.02 \pm 0.51 \\
4.41 \pm 0.41 \\
4.59 \pm 0.68\end{array}$}} & $\mathrm{c}$ & 15.5 \\
\hline & W $182 \mathrm{E}$ & $\mathrm{WD} \times \mathrm{W} 22$ & 0 & & & c & 11.3 \\
\hline & CG 18 & $\mathrm{CG} 7 \times \mathrm{CM} 37$ & -4.5 & & & $\mathrm{c}$ & 18.1 \\
\hline \multicolumn{2}{|l|}{ Mean/tolerant } & & -1.4 & \multicolumn{3}{|c|}{$1.89 \pm 0.12$} & 8.1 \\
\hline \multicolumn{2}{|l|}{ Mean/susceptible } & & 0.8 & \multicolumn{3}{|c|}{$4.33 \pm 0.40$} & 12.8 \\
\hline
\end{tabular}

1 Pedigree selection under ECB infestation at Inra St-Martin de Hinx.

${ }^{2}$ Difference in days with WI82E.

${ }^{3}$ Mean ratings (M) for the nine series of 2-year experiments with their confidence limits (CL) for $P=0.05$, the results of the Friedman test (FT) and the variation coefficient (VC). 


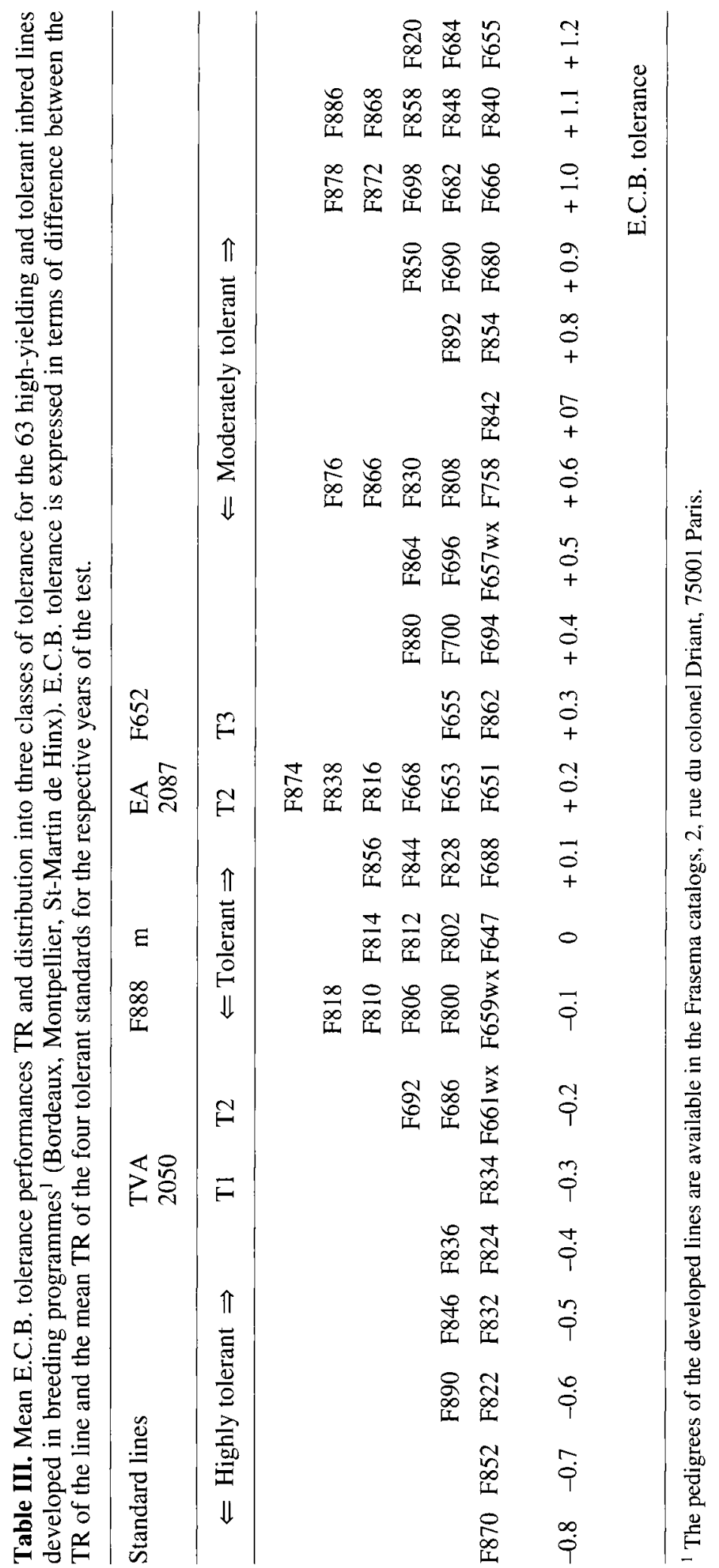


Table IV. Performances of lines originated from North-American dent germplasm.

\begin{tabular}{|c|c|c|c|c|c|c|c|c|c|c|c|}
\hline \multicolumn{5}{|c|}{ Tolerance per se value ${ }^{1}$} & \multicolumn{4}{|c|}{ Reference $\times$ testers hybrid value } & \multicolumn{3}{|c|}{ Gains lines/ref. on testers } \\
\hline $\begin{array}{l}\text { Release } \\
\text { years }\end{array}$ & $\begin{array}{l}\text { Source } \\
\text { lines }\end{array}$ & Tol. & Ref. & Tol. & Ref. $\times$ tester & $\begin{array}{c}\text { Grain } \\
\text { moisture } \\
(\%)\end{array}$ & $\begin{array}{c}\text { Grain } \\
\text { yield } \\
\left(\mathrm{Mg}_{\mathrm{g}} \cdot \mathrm{ha}^{-1}\right)\end{array}$ & $\begin{array}{c}\text { Stalk } \\
\text { lodging } \\
(\%)\end{array}$ & $\begin{array}{c}\text { Grain } \\
\text { moisture }\end{array}$ & $\begin{array}{l}\text { Grain } \\
\text { yield }\end{array}$ & $\begin{array}{c}\text { Stalk } \\
\text { lodging }\end{array}$ \\
\hline \multicolumn{12}{|c|}{ Lancaster } \\
\hline 1990 & F684 & $\mathrm{T} 3$ & $\mathrm{MO} 17$ & $\mathrm{~T} 3$ & $\mathrm{MO} 17 \times \mathrm{B} 73$ & 30 & 117 & 14 & $\mathrm{ME}$ & & \\
\hline \multicolumn{12}{|c|}{ Stiff Stalk } \\
\hline $\begin{array}{l}1994 \\
1995\end{array}$ & $\begin{array}{l}\text { F848 } \\
\text { F876 }\end{array}$ & $\begin{array}{l}\mathrm{T} 3 \\
\mathrm{~T} 3\end{array}$ & $\begin{array}{l}\text { F618 } \\
\text { F618 }\end{array}$ & $\begin{array}{l}\text { S3 } \\
\text { S3 }\end{array}$ & $\begin{array}{l}\mathrm{F} 618 \times \mathrm{MO} 17 \\
\mathrm{~F} 618 \times \mathrm{MBS} 847\end{array}$ & $\begin{array}{l}32 \\
30\end{array}$ & $\begin{array}{l}96 \\
86\end{array}$ & $\begin{array}{r}19 \\
9\end{array}$ & \multicolumn{3}{|c|}{ MHY } \\
\hline \multicolumn{12}{|c|}{ Early dent Wisconsin } \\
\hline 1990 & F686 & $\mathrm{T} 2$ & B73 & $\mathrm{S} 2$ & $\mathrm{~B} 73 \times \mathrm{MO} 17$ & 30 & 117 & 14 & ME & \multirow{3}{*}{\multicolumn{2}{|c|}{ HY }} \\
\hline 1991 & F694 & $\mathrm{T} 3$ & $\mathrm{~F} 2$ & T3 & $\mathrm{F} 2 \times \mathrm{MBS} 847$ & 29 & 99 & 1 & & & \\
\hline 1991 & F700 & $\mathrm{T} 3$ & A654 & S3 & A654 $\times$ MBS 847 & 30 & 104 & 2 & ME & & \\
\hline
\end{tabular}

I Tolerance classes: $\mathrm{T} 1$ = highly folerant, $\mathrm{T} 2$ = tolerant, $\mathrm{T} 3$ = moderately tolerant; susceptibility classes: S3 moderately susceptible, $\mathrm{S} 2$ = susceptible, $\mathrm{S} 1$ = highly susceptible.

2 Gains of performances observed in line $\times$ lester compared to reference $\times$ tester lest-crosses: grain moisture: ME $=$ more early $($ moisture $\leq 120 \%$ of the reference); $\mathrm{LE}=$ less early (moisture $>120 \%$ ), grain yield: HY = higher yield $(\geq 105 \%$ of the reference), MHY $=$ much higher yield $(\geq 110 \%)$ stalk lodging: $\mathrm{MT}=$ more tolerant $(\geq 20 \%$ decreased lodging $): \mathrm{LT}=$ less tolerant $(\geq 20 \%$ increase lodging).

(table $/ I I$ ). To do so, the tolerance of the standards used in the different years of test were studied. In spite of annual variations in the insect-plant relationships (insect survival rate, plant reaction to insect feeding), four tolerant and three susceptible standards presented among them a stability for their ratings and respective rankings, verified by different criteria: confidence limits of the mean, variation coefficient, non-parametric Friedman test (table II). Consequently, tolerance of lines could be expressed (table III) in terms of difference between their own TR and the mean TR of the four tolerant standards for their respective years of test and release.

Second, an analysis was made concerning the responses of different germplasms to breeding. Twelve groups of origins with 44 lines were studied. The lines were arranged according to their genetic origin whatever their years of test. It must be kept in mind that data originate from comparison in individual pairs (the reference line and the tested one) in different years of test. Consequently, the values of the gains obtained by breeding must be considered with caution.

\section{RESULTS AND DISCUSSION}

\subsection{Tolerance of the inbred lines}

As indicated above, the tolerance data of the lines are given in table III. The statistical parameters observed for the tolerant standards were such that the lines were arranged into three classes: highly tolerant T1 ( $\geq$ TVA 2050 ), tolerant T2 including F888 and EA 2087 and moderately tolerant T3 ( $\leq$ F 652).

\subsection{Responses of germplasm sources to breeding}

The results of analysis are given in the tables $I V-V I$ and VII. 
Table V. Performances of lines originating from the European (E) and from Argentina-Europe (AE) germplasms and from their crosses with North America dent germplasm (NA).

\begin{tabular}{|c|c|c|c|c|c|c|c|c|c|}
\hline \multicolumn{5}{|c|}{ Tolerance per se value } & \multicolumn{4}{|c|}{ Reference $\times$ testers hybrid value } & Gains lines/ref. on testers ${ }^{2}$ \\
\hline $\begin{array}{l}\text { Release } \\
\text { years }\end{array}$ & $\begin{array}{l}\text { Source } \\
\text { lines }\end{array}$ & Tol. & Ref. & Tol. & Ref. $\times$ tester & $\begin{array}{l}\text { Grain } \\
\text { moisture } \\
(\%)\end{array}$ & $\begin{array}{c}\text { Grain } \\
\text { yield } \\
\left(\mathrm{Mg} \cdot h \mathrm{a}^{-1}\right)\end{array}$ & $\begin{array}{l}\text { Stalk } \\
\text { lodging } \\
(\%)\end{array}$ & $\begin{array}{l}\text { Grain Grain Stalk } \\
\text { moisture yield lodging }\end{array}$ \\
\hline
\end{tabular}

Euskadian population

$\begin{array}{llllllllll}1991 & \text { F810 } & \text { T2 } & \text { F2 } & \text { T3 } & \text { F2 } \times \text { MBS847 } & 29 & 99 & 1 & \text { MHY }\end{array}$

Early Flint E composite

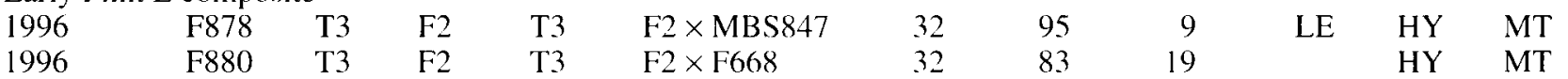

AE Flint composite

\begin{tabular}{|c|c|c|c|c|c|c|c|c|c|c|c|}
\hline 1993 & F651 & $\mathrm{T} 2$ & F492 & S3 & $\mathrm{F} 492 \times \mathrm{MBS} 847$ & 28 & 84 & 10 & & MHY & LT \\
\hline 1993 & F653 & $\mathrm{T} 2$ & $\mathrm{~F} 2$ & $\mathrm{~T} 3$ & F2 $\times$ Early dent & 30 & 76 & 31 & LE & HY & MT \\
\hline 92 & F822 & $\mathrm{T} 1$ & $\mathrm{~F} 2$ & $\mathrm{~T} 3$ & $\mathrm{~F} 2 \times \mathrm{MBS} 847$ & 32 & 98 & 8 & LE & HY & גT \\
\hline 92 & F828 & $\mathrm{T} 2$ & $\mathrm{~F} 2$ & $\mathrm{~T} 3$ & $\mathrm{~F} 2 \times \mathrm{MBS} 847$ & 32 & 98 & 8 & LE & & \\
\hline 92 & F830 & $\mathrm{T} 3$ & $\mathrm{~F} 2$ & $\mathrm{~T} 3$ & $\mathrm{~F} 2 \times \mathrm{MBS} 847$ & 32 & 98 & 8 & LE & HY & \\
\hline 95 & F862 & $\mathrm{T} 3$ & F564 & $\mathrm{T} 2$ & $\mathrm{~F} 2 \times \mathrm{MBS} 847$ & 37 & 104 & 16 & & & \\
\hline 97 & F886 & T3 & F564 & $\mathrm{T} 2$ & $\mathrm{~F} 564 \times \mathrm{F} 692$ & 36 & 92 & 30 & & MHY & \\
\hline & $\mathrm{F} 650$ & $\mathrm{~T} 2$ & F651 & T? & $F 651 \times F 618$ & 29 & 101 & 17 & LE & HY & \\
\hline
\end{tabular}

E Composite $\mathrm{A} \times$ Lancaster

\begin{tabular}{|c|c|c|c|c|c|c|c|c|c|c|}
\hline 1991 & F814 & $\mathrm{T} 2$ & MBS & S3 & MBS $847 \times$ F816 & 22 & 97 & 6 & & $\mathrm{HY}$ \\
\hline 1991 & F816 & $\mathrm{T} 2$ & MBS & S3 & MBS $847 \times$ F816 & 22 & 97 & 6 & LE & MHY \\
\hline 1993 & F836 & $\mathrm{T} 1$ & F492 & S3 & $\mathrm{F} 492 \times \mathrm{MBS} 847$ & 28 & 84 & 10 & ME & MHY \\
\hline
\end{tabular}

E Composite $\mathrm{B} \times \mathrm{SSS}$

\begin{tabular}{|c|c|c|c|c|c|c|c|c|c|c|}
\hline 1991 & $\mathrm{~F} 800$ & $\mathrm{~T} 2$ & $\mathrm{~F} 2$ & $\mathrm{~T} 3$ & $\mathrm{~F} 2 \times$ Early dent & 26 & 89 & 1 & & HY \\
\hline 1993 & F834 & $\mathrm{T} 1$ & A632 & T3 & A $632 \times$ Early dent & 33 & 82 & 29 & & $\mathrm{HY}$ \\
\hline 1994 & F846 & $\mathrm{T} 1$ & F618 & S3 & $\mathrm{F} 618 \times \mathrm{MO} 17$ & 32 & 96 & 19 & ME & \\
\hline 1997 & F665 & $\mathrm{T} 3$ & F618 & S3 & $\mathrm{F} 618 \times \mathrm{MO} 17$ & 30 & 108 & 22 & & HY \\
\hline 1997 & F661 & $\mathrm{T} 2$ & F618 & $\mathrm{S} 3$ & $\mathrm{~F} 618 \times \mathrm{MO} 17$ & 31 & 112 & 16 & & $\mathrm{HY}$ \\
\hline
\end{tabular}

Pool AE + NA

\begin{tabular}{|c|c|c|c|c|c|c|c|c|c|c|c|}
\hline 1995 & F655 & $\mathrm{T} 3$ & F618 & S3 & F618 $\times$ MBS847 & 30 & 86 & 9 & \multicolumn{3}{|c|}{ HY } \\
\hline 1991 & $\mathrm{~F} 802$ & $\mathrm{~T} 2$ & MO17 & T3 & $\mathrm{MO} 17 \times \mathrm{MBS} 847$ & 24 & 99 & 2 & $\mathrm{ME}$ & & \\
\hline 1995 & F864 & $\mathrm{T} 3$ & F598 & $\mathrm{T} 3$ & $\mathrm{~F} 598 \times \mathrm{MBS} 847$ & 35 & 100 & 6 & & & \\
\hline 1995 & F866 & $\mathrm{T} 3$ & F611 & SI & F611 $\times$ MBS847 & 38 & 87 & 19 & ME & MHY & MT \\
\hline
\end{tabular}

1.2 As in table IV.

\subsubsection{Global results}

For agronomic traits, about 40 and $25 \%$, respectively, of the lines presented grain yields higher (HY) $(105 \%)$ and much higher (MHY) $(110 \%)$ than the reference crossed on the same tester. Improvements in stalk lodging tolerance (MT) were observed for $55 \%$ of the lines which exhibit- ed a $20 \%$ decrease in lodging by comparison to the references. The grain moisture at harvest was higher (LE) or lower (ME) than references, respectively, for 18 and $25 \%$ of the lines. In some cases, high gains were simultaneously obtained for the three agronomic criteria ( $F$ 653, F 659 wx, F 822, F 830, F 842, F 850, F 866, F 870, F 878). 
Table VI. Performances of lines originated from backcrosses and single crosses.

\begin{tabular}{|c|c|c|c|c|c|c|c|c|c|c|c|}
\hline \multicolumn{5}{|c|}{ Tolerance per se value $^{1}$} & \multicolumn{4}{|c|}{ Reference $\times$ testers hybrid value } & \multicolumn{3}{|c|}{ Gains lines/ref. on testers ${ }^{2}$} \\
\hline $\begin{array}{l}\text { Release } \\
\text { years }\end{array}$ & $\begin{array}{l}\text { Source } \\
\text { lines }\end{array}$ & Tol. & Ref. & Tol. & Ref. $\times$ tester & $\begin{array}{c}\text { Grain } \\
\text { moisture } \\
(\%)\end{array}$ & $\begin{array}{c}\text { Grain } \\
\text { yield } \\
\left(\mathrm{Mg} \cdot \mathrm{ha}^{-1}\right)\end{array}$ & $\begin{array}{c}\text { Stalk } \\
\text { lodging } \\
(\%)\end{array}$ & $\begin{array}{c}\text { Grain } \\
\text { moisture }\end{array}$ & $\begin{array}{l}\text { Grain } \\
\text { yield }\end{array}$ & $\begin{array}{c}\text { Stalk } \\
\text { lodging }\end{array}$ \\
\hline \multicolumn{12}{|c|}{ Lacaune Flint $\mathrm{F}^{2}{ }^{2} \times$ Early dent NA } \\
\hline 1992 & F818 & $\mathrm{T} 2$ & $\mathrm{~F} 2$ & $\mathrm{~T} 3$ & $\mathrm{~F} 2 \times \mathrm{MBS} 847$ & 32 & 98 & 8 & $\mathrm{ME}$ & & MT \\
\hline 1992 & $\mathrm{~F} 820$ & $\mathrm{~T} 3$ & $\mathrm{~F} 2$ & $\mathrm{~T} 3$ & $\mathrm{~F} 2 \times \mathrm{MBS} 847$ & 32 & 98 & 8 & & MHY & MT \\
\hline 1994 & $\mathrm{~F} 840$ & $\mathrm{~T} 3$ & $\mathrm{~F} 2$ & $\mathrm{~T} 3$ & $\mathrm{~F} 2 \times \mathrm{MBS} 847$ & 35 & 93 & 9 & & HY & \\
\hline 1994 & F842 & $\mathrm{T} 3$ & $\mathrm{~F} 2$ & $\mathrm{~T} 3$ & $\mathrm{~F} 2 \times \mathrm{MBS} 847$ & 35 & 93 & 9 & LE & $\mathrm{HY}$ & MT \\
\hline 1994 & F844 & $\mathrm{T} 2$ & $\mathrm{~F} 2$ & $\mathrm{~T} 3$ & $\mathrm{~F} 2 \times \mathrm{MBS} 847$ & 35 & 93 & 9 & & MHY & MT \\
\hline \multicolumn{12}{|c|}{ Lacaune Flint $F 7^{2} \times$ Early dent NA } \\
\hline 1991 & F696 & $\mathrm{T} 3$ & F7 & S1 & F7 $\times$ MBS847 & 30 & 99 & 3 & & $\mathrm{HY}$ & \\
\hline 1991 & F698 & $\mathrm{T} 3$ & F7 & $\mathrm{S} 1$ & F7 $\times$ MBS 847 & 30 & 99 & 3 & & HY & \\
\hline \multicolumn{12}{|c|}{$\mathrm{F} 255(\mathrm{~T} 3) \times \mathrm{C} 0255(\mathrm{~S} 1)$} \\
\hline 1994 & F852 & $\mathrm{T} 1$ & & & $\mathrm{CO} 255 \times \mathrm{F} 618$ & 35 & 86 & 23 & & & MT \\
\hline
\end{tabular}

${ }^{1}$ Tolerance classes: $\mathrm{T} 1=$ highly tolerant, $\mathrm{T} 2=$ tolerant, $\mathrm{T} 3=$ moderately tolerant; susceptibility classes: $\mathrm{S} 3$ moderately susceptible, S2 = susceptible, $\mathrm{S} 1$ = highly susceptible.

${ }^{2}$ Gains of performances observed in line $\times$ tester compared to reference $\times$ tester test-crosses: grain moisture: $\mathrm{ME}=$ more early (moisture $\leq 120 \%$ of the reference); $\mathrm{LE}=$ less early (moisture $>120 \%$ ), grain yield: $\mathrm{HY}=$ higher yield $(\geq 105 \%$ of the reference), MHY $=$ much higher yield $(\geq 110 \%)$ stalk lodging: MT = more tolerant $(\geq 20 \%$ decreased lodging $) ; \mathrm{LT}=$ less tolerant $(\geq 20 \%$ increase lodging).

Table VII. Performances of lines originated from ECB tolerant or moderately resistant materials.

\begin{tabular}{|c|c|c|c|c|c|c|c|c|c|}
\hline \multicolumn{5}{|c|}{ Tolerance per se value ${ }^{l}$} & \multicolumn{4}{|c|}{ Reference $\times$ testers hybrid value } & Gains lines/ref. on testers ${ }^{2}$ \\
\hline $\begin{array}{l}\text { Release } \\
\text { years }\end{array}$ & $\begin{array}{l}\text { Source } \\
\text { lines }\end{array}$ & Tol. & Ref. & Tol. & Ref. $\times$ tester & $\begin{array}{l}\text { Grain } \\
\text { moisture } \\
(\%)\end{array}$ & $\begin{array}{c}\text { Grain } \\
\text { yield } \\
\left(\mathrm{Mg}^{-1} \mathrm{ha}^{-1}\right)\end{array}$ & $\begin{array}{l}\text { Stalk } \\
\text { lodging } \\
(\%)\end{array}$ & $\begin{array}{c}\text { Grain Grain } \begin{array}{c}\text { Stalk } \\
\text { moisture yield }\end{array} \text { lodging }\end{array}$ \\
\hline
\end{tabular}

Tolerant Synthetic FS 12

$\begin{array}{lllllllllll}1991 & \text { F812 } & \text { T2 } & \text { A632 } & \text { T3 } & \text { A632 } \times \text { MBS847 } & 24 & 99 & 2 & \text { ME } & \\ 1992 & \text { F832 } & \text { T1 } & \text { A632 } & \text { T3 } & \text { A632 } \times \text { Early dent } & 31 & 94 & 21 & & \text { MT } \\ 1995 & \text { F874 } & \text { T2 } & \text { F812 } & \text { T2 } & \text { F812 } \times \text { MO17 } & 29 & 78 & 13 & & \text { MHY }\end{array}$

Polygenic H.T. resistance source

$\begin{array}{llllllllllll}1994 & \text { F850 } & \text { T3 } & \text { F566 } & \text { S2 } & \text { F566 } \times \text { Early dent } & 35 & 79 & 27 & \text { ME } & \text { HY } & \text { MT } \\ 1995 & \text { F868 } & \text { T3 } & \text { F566 } & \text { S2 } & \text { F566 } \times \text { F618 } & 31 & 84 & 13 & & & \\ 1995 & \text { F870 } & \text { T1 } & \text { F566 } & \text { S2 } & \text { F566 } \times \text { MBS847 } & 30 & 79 & 12 & \text { ME } & \text { MHY } & \text { MT }\end{array}$

1 Tolerance classes: $\mathrm{T} 1$ = highly tolerant, $\mathrm{T} 2$ = tolerant, $\mathrm{T} 3=$ moderately tolerant; susceptibility classes: S3 moderately susceptible, $\mathrm{S} 2$ = susceptible, $\mathrm{S} 1=$ highly susceptible.

${ }^{2}$ Gains of performances observed in line $\times$ tester compared to reference $\times$ tester test-crosses: grain moisture: $\mathrm{ME}=$ more early (moisture $\leq 120 \%$ of the reference); $\mathrm{LE}=$ less early (moisture $>120 \%$ ), grain yield: $H Y=$ higher yield ( $\geq 105 \%$ of the reference), MHY $=$ much higher yield $(\geq 110 \%)$ stalk lodging: $\mathrm{MT}=$ more tolerant $(\geq 20 \%$ decreased lodging $) ; \mathrm{LI}=$ less tolerant $(\geq 20 \%$ increase lodging). 
Globally the E.C.B. tolerance for $68 \%$ of the lines were higher than the tolerance of the references. For about $2 / 3$ of these lines, the higher level of tolerance was correlated with an improvement of their yields when they are crossed on the same tester than the reference: for instance, the lines F 810, F 822, F $844>$ F2 in the test crosses with MBS 847; the lines F 655, F 876 and F 665, F 661 WX > F 618 in the test crosses with MBS 847 and MO 17, respectively.

\subsubsection{Variations according the germplasms}

The lines obtained from the North-American dent sources were generally moderately tolerant (table IV). On the contrary, a good half of the lines derivated from European (E) and ArgentinianEuropean (AE) composites were tolerant or highly tolerant. Moreover, they are more tolerant to stalk lodging and higher yielding, but generally less early than the references F 2 and F 564 (table V).

The progenies of the European composites crossed by the American sources Lancaster and Stiff Stalk Synthetic were as a whole tolerant or highly tolerant and, for the same level of earliness, generally more stalk lodging tolerant and higher yielding than the references MBS 847, F 618 or MO 17 (table V).

These results have shown that it is probably easier to obtain $\mathrm{T} 1$ and $\mathrm{T} 2$ tolerant lines from the generally flint European sources than from the dent $\mathrm{N}$. American material. Is this property linked to the flint type or rather favoured by the more than 450 years of insect selection pressure on the material introduced into the Old World from where the borer originates?

Moreover, back-crosses by well known flint French lines of early dent N. American sources have allowed the obtention of higher yielding lines than $F 2$ and $F 7$, with a better tolerance than the highly susceptible F 7 for F 696 and F 698. In the progeny of a susceptible single cross, transgression to tolerance was observed in the F 852 line (table VI). In addition, table VII exhibited improvements for different criteria in synthetic materials already known for tolerance.

\section{GENERAL COMMENTS AND CONCLUSION}

Before the beginning of our present breeding programme, among the three interrelated bases of insect resistance defined as preference, antibiosis and tolerance [13], American maize breeders set out to discover antibiosis characters increasing young E.C.B. larvae mortality in their leaf and sheath collar feeding. They used high levels of infestation associated with high selection pressure and obtained a large number of leaf-feeding resistant lines. Painter (op. cit.) stated that "the insect must always be the final judge of whether a new variety, selection or hybrid is really resistant". For our part, this idea associated with the concept of tolerance has governed our approach based on the whole insect-plant relationship until maize maturity. In 1976, our breeding project was new and original, first in its objective (developing from European germplasm lines combining E.C.B. tolerance with high agronomic value) and second in its method of evaluating tolerance (low infestation rate in order to appreciate small differences in susceptibility, choice of suitable standards, adjustment of data to earliness) and agronomic value both by breeders and entomologists.

In studies carried out in North America, the criteria of tolerance and yield in breeding for resistance have appeared only recently $[10,12,17]$. However, the present selection for standability and reduction of yield losses for infestations after anthesis belongs mainly to tolerance. The American criteria for cavity counts or length of tunnels are similar to the percentage of damaged internodes correlated with the visual tolerance ratings used in our programmes.

At present, in different countries, the breeding programmes for E.C.B resistance use tropical sources: for instance, development of the MO-2 ECB 2 [4] and of F 888 from ETO (M) C9 in France, CIMMYT programme for multiple borer resistance [5]. Moreover, back-crosses are used in transferring resistance or tolerance characters to susceptible elite lines. For our part, a back-cross breeding programme $\mathrm{DE} 811 \times \mathrm{F} 618$ is in pro- 
gress. The studies of mapping and characterisation of QTLs affecting resistance of lines derived from the cross B73 susceptible $\times$ B52 resistant with aid of RFLPs conducted at Ames University (Iowa) and Hohenheim University (Germany) (see for instance Schön et al. [16]) should make it possible to develop inbred lines with high hybrid values and E.C.B. tolerance.

In conclusion, over the course of 16 years, 63 flint and dent inbred lines, adapted to European conditions with high yielding ability and appreciable levels of E.C.B tolerance have been developed and released to maize breeders. They can be considered to contribute to an economical and ecological way of controlling the E.C.B.

Acknowledgements: The authors are indebted to the Inra technicians for producing the maize seeds, for taking up cultural practices and field observations as well as to the experimenters of the Geli network.

\section{REFERENCES}

[1] Anglade P., Hadzistevic D., International cooperative study of resistance of several European inbred lines of maize to the ECB. E.P.P.O. Pub. 54 (1970) $57-62$.

[2] Anglade P., Benas G., Cangardel H., Goix J., Présence de la pyrale sur les cultures de maïs du bassin Parisien, Rev. Zool. Agr. 65 (1966) 126-127.

[3| Anglade P., Gouesnard B., Boyat A. Panouillé A., Effects of multitrait recurrent selection for ECB tolerance and for agronomic traits in FS 12 maize synthetic, Maydica 41 (1996) 97-104.

[4] Barry B.D., Darrah L.L., Development of germplasm with resistance to the European corn borer, in: Mihm J.A. (Ed.), Insect Resistant Maize. Recent Advances and Utilization, Proc. Int. Symp. C.I.M.M.Y.T., Mexico, 1994, pp. 217-220.

15] Bergvinson D.J., Arnason J.T., Mihm J.A., Jewell D.C., Phytochemical basis for multiple borer resistance in maize, in: Nihm J.A. (Ed.), Insect Resistant Maize. Recent Advances and Utilization, Proc. Int. Symp. C.I.M.M.Y.T., Mexico, 1994, pp. 82-90.

|6| Guthrie W.D., Dicke F.F., Resistance of inbred lines of dent corn to leaf feeding by 1 st-brood European corn borers, Ia. St. J. Sci. 46 (1972) 339-357.
[7] Guthrie W.D., Huggans J.L.,Chatterji S.M., Sheath collar feeding resistance to the second brood European corn borer in six inbred lines of dent corn, Ia. St. J. Sci. 44 (1970) 65-83.

[8] Hawlitsky N., Étude de la biologie de la pyrale du maïs, Ostrinia nubilalis $\mathrm{Hbn}$, en région parisienne durant quatre années et de recherche d'éléments prévisionnels du début de ponte, Acta oecol./Oecologia Appl. 7 (1986) 47-68.

[9] Kaan F., Anglade P., Boyat A., Panouillé A., La résistance à la Pyrale $O$. nubilalis $\mathrm{Hbn}$., dans un diallèle de 14 lignées précoces de maïs, Agronomie 3 (1983) 507-512.

[10] Klenke J.R., Russel W.A., Guthrie W.D., Smith O.S., Inbreeding depression and gene frequency changes for agronomic traits in corn synthetic selected for resistance to European corn borer, J. Agric. Entomol. 5 (1988) 225-233.

[11] Klun J.A., Guthrie W.D., Hallauer A.R., Russel W.A., Genetic nature of the concentration of 2,4-dihydroxy-7-methoxy $2 \mathrm{H}-1.4$ benzoxazin $-3(4 \mathrm{H})$ - one and resistance to the European corn borer in a diallel set of eleven maize inbreds, Crop Sci. 10 (1970) 87-90.

[12] Nyhus K.A., Russel W.A., Guthrie W.D., Changes in agronomic traits associated with recurrent selection in two maize synthetics, Crop Sci. 29 (1989) 269-275.

[13] Painter R.H., Insect Resistance in Crop Plants, C. Millan, New York, 1951.

[14] Russel W.A., Guthrie W.D., Grindeland R.L., Breeding for resistance in maize to first and second broods of the European corn borer, Crop Sci. 14 (1974) $725-727$.

[15] Sagers J., Edwards M., Bolan B., Wang A., Mettler I., Barret L., Garret C., Mies D., Developing maize with resistance to European Corn Borer, in: Mihm J.A. (Ed.), Insect Resistant Maize. Recent Advances and Utilization, Proc. Int. Symp. C.I.M.M.Y.T., Mexico, 1994, pp. 193-171.

[16] Schön C.C., Lee M. Melchinger A.E., Guthrie W.D., Woodman W.L., Mapping and characterization of quantitative trait loci affecting resistance against second generation European corn borer in maize with the aid of RFLPs. Heredity 70 (1993) 648-659.

[17] Widstrom N.W, Bondari K., Mc Millan W.W., Hybrid performance among maize populations selected for resistance to insects, Crop Sci. 32 (1992) 85-89. 